\title{
Probabilistic Selection of High-redshift Quasars with Subaru/Hyper Suprime-Cam Survey
}

\author{
Masafusa Onoue ${ }^{1,2}$ \\ ${ }^{1}$ Dept. of Astronomical Science, SOKENDAI (The Graduated University for Advanced \\ Studies), Mitaka, Tokyo 181-8588, Japan \\ email: masafusa.onoue@nao.ac.jp \\ ${ }^{2}$ Optical and Infrared Astronomy Division, National Astronomical Observatory of Japan, \\ Mitaka, Tokyo 181-8588, Japan
}

\begin{abstract}
High-redshift quasars are an important probe of the distant Universe. They enable observational studies of the early growth of supermassive blackholes, cosmic reionization, chemical enrichment of host galaxies, and so on. Through pioneering optical and near-infrared wide-area surveys such as the SDSS and the VIKING Survey, about one hundred quasars have been found at $z>6$ (e.g., Fan et al. (2006b), Venemans et al. (2013)). However, its current small sample size and the fact that most of them are the most luminous $\left(M_{1450} \lesssim-24\right)$ population in this epoch prevents one from constraining statistics on high-redshift quasars, namely quasar luminosity function (QLF), and redshift evolution of IGM neutral fraction. Thus, discovery of large number of $z>6$ quasars, especially low-luminous or $z>7$ quasars, is highly desired for further understanding of the early universe.

We are now starting a new ground-breaking survey of high-redshift $(z>6)$ quasars using the exquisite imaging data provided by the Hyper Suprime-Cam (HSC) Subaru Strategic Program (SSP) Survey. Thanks to its extremely wide coverage and its high sensitivity thorough five optical bands (1,400 deg ${ }^{2}$ to the depth of $r \sim 26$ in HSC-Wide layer), it is one of the most powerful contemporary surveys that makes it possible for us to increase the number of $z>6$ quasars by almost an order of magnitude, i.e., 300 at $z \sim 6$ and 50 at $z \sim 7$, based on the current estimate of the QLF at $z>6$ by Willott et al. (2010b).

One of the biggest challenges in $z>6$ quasar candidate selection is contamination of Galactic brown dwarfs, which have the same point-like appearance as and similarly red colors to the quasars. To overcome this issue and maximize the selection efficiency, we apply a double-layered approach to the HSC survey products, namely combination of two probabilistic selections: SEDfitting and Bayesian selection. In particular, we have developed a template SED fitting method optimized to high-redshift quasars selection. Its application with 27 photometric bands to the COSMOS quasars at $3<z<5$ have shown that almost all of the known quasars are correctly classified with small dispersion $\sigma_{|\Delta z| /(1+z)}=0.01$ and as low as $\eta=2.5 \%$ outlier rate. At present, the HSC survey have successfully covered $\sim 100 \mathrm{deg}^{2}$ of HSC-Wide area in full colors. From the first-year data products, we have already started $z>6$ quasar selection, and it is expected that the first HSC quasar discovery will be in the near future.
\end{abstract}

Keywords. cosmology: observation - quasars: general - surveys

\section{References}

Fan, X. et al. 2006b, AJ, 132, 117

Venemans, B. P. et al. 2013, ApJ, 779, 24

Willott, C. J., Delorme, P., Reyle, C., et al. 2010b, AJ, 139, 906 